\title{
Gravitational waves from a Weyl-Integrable manifold: a new formalism.
}

\author{
${ }^{1}$ Jesús Martín Romero, ${ }^{1,2}$ Mauricio Bellini and ${ }^{3}$ José Edgar Madriz Aguilar \\ ${ }^{1}$ Departamento de Física, Facultad de Ciencias Exactas y Naturales \\ Universidad Nacional de Mar del Plata, \\ Funes 3350, C.P. 7600, Mar del Plata, Argentina \\ ${ }^{2}$ Instituto de Investigaciones Físicas de Mar del Plata (IFIMAR) \\ Consejo Nacional de Investigaciones Científicas y Técnicas (CONICET), Argentina. \\ E-mail: jesusromero@conicet.gov.ar,mbellini@mdp.edu.ar \\ ${ }^{3}$ Departamento de Matemáticas, Centro Universitario \\ de Ciencias Exactas e ingenierías (CUCEI), \\ Universidad de Guadalajara (UdG), \\ Av. Revolución 1500 S.R. 44430, Guadalajara, Jalisco, México. \\ E-mail:madriz@mdp.edu.ar,edgar.madriz@red.cucei.udg.mx
}

\begin{abstract}
We study the variational principle over an Hilbert-Einstein like action for an extended geometry taking into account torsion and non-metricity. By extending the semi-Riemannian geometry, we obtain an effective energy-momentum tensor which can be interpreted as physical sources. As an application we develop a new manner to obtain the gravitational wave equations on a Weylintegrable manifold taking into account the non-metricity and non-trivial boundary conditions on the minimization of the action, which can be identified as possible sources for the cosmological constant and provides two different equations for gravitational waves. We examine gravitational waves in a pre-inflationary cosmological model.
\end{abstract}

PACS numbers: 04.50. Kd, 04.20.Jb, 11.10.kk, 98.80.Cq 
Torsion, nonmetricity, Weyl-Integrable gravity, gravitational waves, inflation.

\section{INTRODUCTION}

In the standard treatment to minimize the action, when a manifold has a boundary $\partial \mathcal{M}$, the action should be supplemented by a boundary term, so that the variational principle to be well-defined [1, 2]. However, this is not the only manner to study this problem. As was recently demonstrated[3], there is another way to include the flux around a hypersurface that encloses a physical source without the inclusion of another term in the Hilbert-Einstein (HE) action. This treatment imposes a constraint on the dynamics obtained by varying the $\mathrm{EH}$ action. In that paper was demonstrated that the non-zero flux of the vector metric fluctuations through the closed 3D Gaussian-like hypersurface, is responsible for the gaugeinvariance of gravitational waves. In present paper we are dealing with the variational principle over a Hilbert-Einstein like action, using an extended geometry with torsion and non-metricity, from which we obtain an effective energy-momentum tensor with sources in the torsion and the non-metricity. It can be viewed in a Riemannian geometry as a describing an effective stress tensor that represents a geometrically induced matter. Additionally, we develop a new manner to obtain gravitational waves on a Weyl-integrable manifold, which has non-metricity and nontrivial boundary terms included.

The paper is organised as follows: in the following section we shall study the general formalism. In Sect. IV we examine the formalism in absence of torsion, taking into account purely Weylian contributions. In Sect. V we deal only with the contributions due to boundary terms. In Sect. VI we examine an example in which massless gravitons are emitted during a pre-inflationary epoch of the universe. Finally, in Sect. VII we develop some final remarks.

\section{GENERAL FORMALISM}

We consider the variational principle in presence of torsion and non-metricity in an Hilbert-Einstein action. We shall start by considering an action in an extended geometry (i.e. a non-Riemannian manifold) conformed by a gravitational sector without the presence of matter in such generalized geometry. The Hilbert-Einstein action was extensively studied 
in Riemannian geometry [4], but we shall deal with an extended geometry:

$$
\mathcal{S}=\frac{1}{2 \kappa} \int_{V} d^{4} x \sqrt{-g} \mathcal{R}
$$

where $V$ denotes the volume of a spacetime manifold featured by a non-metricity [5], [6] and a general torsion [5], [7]. Furthermore, $g$ is the determinant of the metric tensor $g_{\alpha \beta}$, the gravitational coupling is denoted by $\kappa=8 \pi G$ and $\mathcal{R}=g^{\alpha \beta} R_{\alpha \beta}$ is the scalar curvature. For a coordinate basis of the tangent space $\left\{\partial_{\sigma}\right\}$, the components of the Riemann tensor are given by

$$
R_{\beta \mu \nu}^{\alpha}=\Gamma_{\beta \nu, \mu}^{\alpha}-\Gamma_{\beta \mu, \nu}^{\alpha}+\Gamma_{\beta \nu}^{\sigma} \Gamma_{\sigma \mu}^{\alpha}-\Gamma_{\beta \mu}^{\sigma} \Gamma_{\sigma \nu}^{\alpha}
$$

where the symbols $\Gamma_{\mu \nu}^{\alpha}$ denote the coordinate components for a generalized connection defined by [5], [8]

$$
\nabla_{\partial_{\alpha}} \partial_{\beta}=\Gamma_{\beta \alpha}^{\epsilon} \partial_{\epsilon}
$$

These components can be written in the general form

$$
\Gamma_{\mu \nu}^{\sigma}=\left\{\begin{array}{l}
\sigma \\
\mu \nu
\end{array}\right\}+K_{\mu \nu}^{\sigma}
$$

where $\left\{\begin{array}{c}\gamma \\ \alpha \beta\end{array}\right\}$ are the components of the usual Riemannian connection (the second kind Christoffel symbols) and $K_{\mu \nu}^{\sigma}$ is a contortion tensor due to torsion and non-metricity, defined by [9]

$$
K_{\mu \nu}^{\sigma}=-\frac{g^{\beta \sigma}}{2}\left\{\tau_{\nu \beta}^{\alpha} g_{\mu \alpha}+\tau_{\mu \beta}^{\alpha} g_{\alpha \nu}-\tau_{\nu \mu}^{\alpha} g_{\alpha \beta}+N_{\mu \beta \nu}+N_{\beta \nu \mu}-N_{\mu \nu \beta}\right\}
$$

such that $\tau_{\mu \nu}^{\alpha}$ and $N_{\alpha \beta \gamma}$ are respectively the torsion and the non-metricity tensors. For a coordinate basis, they are

$$
\begin{aligned}
\tau_{\mu \nu}^{\alpha} & =\Gamma_{\mu \nu}^{\alpha}-\Gamma_{\nu \mu}^{\alpha}, \\
N_{\alpha \beta \gamma} & =g_{\beta \gamma ; \alpha},
\end{aligned}
$$

where the semicolon denotes the covariant derivative defined in terms of the $\Gamma$ connection (i.e. defined on the extended manifold).

Now, in order to derive the dynamical equations for gravitational waves on this general space-time manifold in a novel and consistent manner, we shall use a variational procedure. The variation of the action (11) leaves to the expression

$$
\delta[\sqrt{-g} \mathcal{R}]=\sqrt{-g}\left[\delta g^{\alpha \beta} G_{\alpha \beta}+g^{\alpha \beta} \delta R_{\alpha \beta}\right]
$$


where $G_{\alpha \beta}=R_{\alpha \beta}-(1 / 2) \mathcal{R} g_{\alpha \beta}$ is a generalization of the Einstein tensor, due to the fact that it is calculated in terms of the $\Gamma$ connection (44). Therefore, it is easy to show that the generalized Einstein tensor contains contributions associated with both tensors, torsion and non-metricity. The last term between brackets of the equation (8) can be written as a generalized Palatini's identity in the form

$$
g^{\alpha \beta} \delta R_{\alpha \beta}=W_{; \mu}^{\mu}-g_{; \mu}^{\alpha \beta} W_{\alpha \beta}^{\mu}-\frac{1}{2} g^{\alpha \beta}\left(\delta \Gamma_{\sigma \beta}^{\mu} \tau_{\alpha \mu}^{\sigma}+\delta \Gamma_{\sigma \alpha}^{\mu} \tau_{\beta \mu}^{\sigma}\right)
$$

where $W^{\mu}=g^{\alpha \beta} W_{\alpha \beta}^{\mu}$. Here, we have introduced the auxiliary tensor $W_{\alpha \beta}^{\mu}$ defined by

$$
W_{\alpha \beta}^{\mu}=\delta \Gamma_{\alpha \beta}^{\mu}-\delta \Gamma_{\sigma \beta}^{\sigma} \delta_{\alpha}^{\mu}
$$

Inserting (99) in (8), and using the identity $g_{; \mu}^{\alpha \nu}=-g^{\beta \nu} g^{\alpha \sigma} N_{\sigma \beta \mu}$, we obtain the variation of the gravitational sector of the action (1)

$\delta \mathcal{S}=\int_{V} d^{4} x \sqrt{-g} G_{\alpha \beta} \delta g^{\alpha \beta}+\int_{V} d^{4} x \sqrt{-g} W_{; \mu}^{\mu}+\int_{V} d^{4} x \sqrt{-g} N_{\mu \alpha \beta} W^{\beta \mu \alpha}-\frac{1}{2} \int_{V} d^{4} x \sqrt{-g} \zeta_{\alpha \beta} g^{\alpha \beta}$,

where $\zeta_{\alpha \beta}$ is an auxiliary tensor field, given by

$$
\zeta_{\alpha \beta}=\delta \Gamma_{\sigma \beta}^{\mu} \tau_{\alpha \mu}^{\sigma}+\delta \Gamma_{\sigma \alpha}^{\mu} \tau_{\beta \mu}^{\sigma}
$$

The third and fourth integrals in (11), are respectively related to the presence of nonmetricity and torsion. On the other hand, the second integral in (11) can be reduced to a 3D hypersurface integral, in virtue of the Stokes Theorem:

$$
\int_{V} d^{4} x \sqrt{-g} W_{; \mu}^{\mu}=\int_{\partial V} d^{3} x \sqrt{-g} W^{\mu} n_{\mu}
$$

where $n_{\mu}$ is a vector field which is normal to the hypersurface $\partial V$. It is usual in the literature to suppose that the surface integral must be neglected when the radius of $\partial V$ is large enough to impose that the field $W^{\mu} \rightarrow 0$ in such limit, or when $W^{\mu}$ is tangent to $\partial V$, that is, when $W^{\mu}$ satisfies the relation $W^{\mu} n_{\mu}=0$. In this paper we shall adopt a different path, and we shall see that the 3D hypersurface term is a source for the cosmological constant [3]. From the expression (13), can be noticed that the term $W_{; \nu}^{\mu}=g_{; \nu}^{\alpha \beta} W_{\alpha \beta}^{\mu}+g^{\alpha \beta} W_{\alpha \beta ; \nu}^{\mu} \neq g^{\alpha \beta} W_{\alpha \beta ; \nu}^{\mu}$ has contributions of non-metricity. This implies that, if we drop this term, the new contribution is not very important. However, when we neglect such boundary term, we must be careful with non-metricity. 
We must notice that the Einstein tensor in (11) can be written as a Riemannian part, plus a non-Riemannian one, in the form

$G_{\alpha \beta}=\bar{G}_{\alpha \beta}+K_{\mu \beta \mid \alpha}^{\mu}-K_{\alpha \beta \mid \mu}^{\mu}+K_{\mu \beta}^{\nu} K_{\nu \alpha}^{\mu}-K_{\alpha \beta}^{\nu} K_{\nu \mu}^{\mu}--\frac{g^{\sigma \gamma}}{2}\left(K_{\mu \gamma \mid \sigma}^{\mu}-K_{\sigma \gamma \mid \mu}^{\mu}+K_{\mu \gamma}^{\nu} K_{\nu \sigma}^{\mu}-K_{\sigma \gamma}^{\nu} K_{\nu \mu}^{\mu}\right) g_{\alpha \beta}$.

In this expression, the bar in $\bar{G}_{\alpha \beta}$ indicates that the Einstein tensor is calculated with the Levi-Civita connections. The symbol "|" denotes the Riemannian covariant derivative. It follows from (14) that when the non-metricity and the torsion vanish. The Einstein tensor in the first integral of (11), simply reduces to the usual Einstein tensor calculated with the Levi-Civita connections.

Now, using the equations (4) and (5), the auxiliary tensor $W_{\alpha \beta}^{\sigma}$ and the vector field $W^{\sigma}$, can be written as

$$
\begin{aligned}
W_{\alpha \beta}^{\mu}= & {\left[\frac{g^{\lambda \mu}}{2}\left\{\delta g_{\alpha \lambda, \beta}+\delta g_{\lambda \beta, \alpha}-\delta g_{\beta \alpha, \lambda}-\tau_{\beta \lambda}^{\rho} \delta g_{\alpha \rho}-\tau_{\alpha \lambda}^{\rho} \delta g_{\rho \beta}\right\}\right.} \\
- & \frac{\delta g^{\lambda \mu}}{2}\left\{g_{\alpha \lambda, \beta}+g_{\lambda \beta, \alpha}-g_{\beta \mu, \lambda}-\tau_{\beta \lambda}^{\rho} g_{\alpha \rho}-\tau_{\alpha \lambda}^{\rho} g_{\rho \beta}-N_{\alpha \lambda \beta}-N_{\lambda \beta \alpha}+N_{\beta \alpha \lambda}\right\} \\
- & \left.\frac{g^{\lambda \sigma}}{4}\left(\delta g_{\lambda \sigma, \beta} \delta_{\alpha}^{\mu}+\delta g_{\lambda \sigma, \alpha} \delta_{\beta}^{\mu}\right)+\frac{\delta g^{\lambda \sigma}}{4}\left(g_{\lambda \sigma, \beta} \delta_{\alpha}^{\mu}+g_{\lambda \sigma, \alpha} \delta_{\beta}^{\mu}-N_{\lambda \sigma \beta} \delta_{\alpha}^{\mu}-N_{\lambda \sigma \alpha} \delta_{\beta}^{\mu}\right)\right] \\
+ & {\left[\frac{g^{\lambda \mu}}{2} \tau_{\beta \alpha}^{\rho} \delta g_{\rho \lambda}-\frac{g^{\lambda \mu}}{2} \tau_{\beta \alpha}^{\rho} g_{\rho \lambda}-\frac{g^{\lambda \sigma}}{4}\left(\delta g_{\lambda \sigma, \beta} \delta_{\alpha}^{\mu}-\delta g_{\lambda \sigma, \alpha} \delta_{\beta}^{\mu}\right)\right.} \\
+ & \left.\frac{\delta g^{\lambda \sigma}}{4}\left(g_{\lambda \sigma, \beta} \delta_{\alpha}^{\mu}-g_{\lambda \sigma, \alpha} \delta_{\beta}^{\mu}+N_{\lambda \sigma \beta} \delta_{\alpha}^{\mu}-N_{\lambda \sigma \alpha} \delta_{\beta}^{\mu}\right)\right] . \\
W^{\mu}= & g^{\alpha \beta}\left[\frac{g^{\lambda \mu}}{2}\left\{\delta g_{\alpha \lambda, \beta}+\delta g_{\lambda \beta, \alpha}-\delta g_{\beta \alpha, \lambda}-\tau_{\beta \lambda}^{\rho} \delta g_{\alpha \rho}-\tau_{\alpha \lambda}^{\rho} \delta g_{\rho \beta}\right\}\right. \\
& -\frac{\delta g^{\lambda \mu}}{2}\left\{g_{\alpha \lambda, \beta}+g_{\lambda \beta, \alpha}-g_{\beta \alpha, \lambda}-\tau_{\beta \lambda}^{\rho} g_{\alpha \rho}-\tau_{\alpha \lambda}^{\rho} g_{\rho \beta}-N_{\alpha \lambda \beta}-N_{\lambda \beta \alpha}\right. \\
& \left.+N_{\beta \alpha \lambda}\right\}-\frac{g^{\lambda \sigma}}{4}\left(\delta g_{\lambda \sigma, \beta} \delta_{\alpha}^{\mu}+\delta g_{\lambda \sigma, \alpha} \delta_{\beta}^{\mu}\right)+\frac{\delta g^{\lambda \sigma}}{4}\left(g_{\lambda \sigma, \beta} \delta_{\alpha}^{\mu}+g_{\lambda \sigma, \alpha} \delta_{\beta}^{\mu}\right. \\
& \left.\left.-N_{\lambda \sigma \beta} \delta_{\alpha}^{\mu}-N_{\lambda \sigma \alpha} \delta_{\beta}^{\mu}\right)\right] .
\end{aligned}
$$

Notice that the term in the first bracket of the expression (15) is the symmetric part of $W_{\alpha \beta}^{\mu}$, while that the term in the second bracket is the antisymmetric part of $W_{\alpha \beta}^{\mu}$, which do not contribute in $W^{\mu}$.

\section{THE TORSIONLESS CASE}

The torsion contribution was historically linked to spin matter[10, 11], or magnetic monopoles [12]. This is a controversial topic that deserves a rigorous treatment, which goes 
beyond the scope of this work and we shall study in a further work. For this reason, in this section we shall study the case of a space-time manifold equipped with non-metricity and without torsion. Under these considerations, the equation (11) reduces to

$$
\delta S=\int_{V} d^{4} x \sqrt{-g}\left(G_{\alpha \beta} \delta g^{\alpha \beta}+W_{; \mu}^{\mu}+N_{\mu \alpha \beta} W^{\beta \mu \alpha}\right),
$$

where we are taking into account a boundary condition in which the 3D hypersurface integral term in (11) is nonzero. In the absence of matter sources, we can associate the geometrical vacuum with a physical one, through the introduction of a dynamical cosmological constant term, in the form

$$
W_{; \mu}^{\mu}+N_{\alpha \beta \mu} W^{\mu \alpha \beta}=\Lambda(x) g_{\alpha \beta} \delta g^{\alpha \beta}
$$

Here, $\Lambda(x)$ is a function of the proper time on the manifold defined by the connections (4), and can play the role of a dynamical cosmological constant with two sources: the surfaceassociated term and the non-metricity term of (17). We must see two separated contributions to the cosmological constant

$$
\begin{gathered}
N_{\alpha \beta \mu} W^{\mu \alpha \beta}=\Lambda_{1}(x) g_{\alpha \beta} \delta g^{\alpha \beta}, \\
W_{; \mu}^{\mu}=\Lambda_{2}(x) g_{\alpha \beta} \delta g^{\alpha \beta},
\end{gathered}
$$

with $\Lambda(x)=\Lambda_{1}(x)+\Lambda_{2}(x)$. Hence the equation (17) becomes

$$
\delta S=\int_{V} d^{4} x \sqrt{-g}\left(G_{\alpha \beta}+\Lambda(x) g_{\alpha \beta}\right) \delta g^{\alpha \beta} .
$$

By imposing the condition that the action is an extreme: $\delta S=0$, we obtain

$$
R_{\alpha \beta}-\frac{1}{2} R g_{\alpha \beta}+\Lambda g_{\alpha \beta}=0
$$

which correspond to the extended Einstein field equations in vacuum where the Ricci tensor $R_{\alpha \beta}$ and the scalar curvature $R$, are both calculated with the affine connections (44), without torsion. Using (14), the equations (22) can be written as

$$
\bar{R}_{\alpha \beta}-\frac{1}{2} \bar{R} g_{\alpha \beta}+\Lambda g_{\alpha \beta}=k \bar{T}_{\alpha \beta}
$$

where $\bar{T}_{\alpha \beta}$ is a geometrical tensor which must be interpreted as the effective energymomentum tensor for a Riemannian geometry, but geometrically induced by the nonmetricity of the manifold

$k \bar{T}_{\alpha \beta}=-K_{\mu \beta \mid \alpha}^{\mu}+K_{\alpha \beta \mid \mu}^{\mu}-K_{\mu \beta}^{\sigma} K_{\sigma \alpha}^{\mu}+K_{\alpha \beta}^{\sigma} K_{\sigma \mu}^{\mu}+\frac{1}{2} g^{\lambda \gamma}\left(K_{\mu \gamma \mid \lambda}^{\mu}-K_{\lambda \gamma \mid \mu}^{\mu}+K_{\mu \gamma}^{\sigma} K_{\sigma \lambda}^{\mu}-K_{\lambda \gamma}^{\sigma} K_{\sigma \mu}^{\mu}\right) g_{\alpha \beta}$. 
In absence of torsion, the effective contortion tensor $K_{\mu \nu}^{\sigma}$ is due exclusively by the nonmetricity contribution:

$$
K_{\mu \nu}^{\sigma}=-\frac{1}{2} g^{\lambda \sigma}\left(N_{\mu \lambda \nu}+N_{\lambda \nu \mu}-N_{\mu \nu \lambda}\right)
$$

Physically we can interpret the equations (23) as the Einstein field equations with a cosmological constant term and a geometrically induced tensor $\bar{T}_{\mu \nu}$, playing the role of an energy-momentum tensor associated with matter sources. This is a kind of induced matter theory where matter has a geometrical origin, but in this case it is not induced by a foliation on an extra-dimension, but on the non-metricity of the Weylian connection. One problem with the non-metricity is related with the integrability of the space-time. In simple words, non-metricity leads to the atomic second clock effect. However, one geometry with non-metricity free of this problem is the well known Weyl-Integrable geometry. In the next section we will consider this kind of space-time geometry.

\section{WEYL-INTEGRABLE MANIFOLD AND GRAVITATIONAL WAVES FROM $\Lambda_{1}$}

We shall focus in this section on the particular case of a Weyl-Integrable non-metricity [13]. The non-metricity tensor $N_{\sigma \mu \nu}$ and the non-metricity contortion tensor $K_{\alpha \beta}^{\mu}$, are given by the expressions

$$
\begin{aligned}
& N_{\sigma \mu \nu}=g_{\mu \nu ; \sigma}=\varphi_{, \sigma} g_{\mu \nu}, \\
& K_{\alpha \beta}^{\mu}=g_{\alpha \beta} \varphi^{, \mu}-\delta_{\alpha}^{\mu} \varphi_{, \beta}-\delta_{\beta}^{\mu} \varphi_{, \alpha},
\end{aligned}
$$

where $\varphi(x)$ is a scalar field known in the literature as the Weyl scalar field.

The field equations (23) are still valid for this particular non-metricity, and the equation (19) now reads

$$
W^{\alpha} \varphi_{, \alpha}=\Lambda_{1} g_{\alpha \beta} \delta g^{\alpha \beta}
$$

where $W^{\mu}$ is given by

$$
\begin{aligned}
W^{\mu} & =g^{\alpha \beta}\left[\frac{g^{\lambda \mu}}{2}\left\{\delta g_{\alpha \lambda, \beta}+\delta g_{\lambda \beta, \alpha}-\delta g_{\beta \alpha, \lambda}\right\}-\frac{\delta g^{\lambda \mu}}{2}\left[g_{\alpha \lambda, \beta}+g_{\lambda \beta, \alpha}-g_{\beta \alpha, \lambda}-g_{\alpha \lambda} \varphi_{, \beta}-g_{\lambda \beta} \varphi_{, \alpha}\right.\right. \\
& \left.\left.+g_{\beta \alpha} \varphi, \lambda\right]-\frac{g^{\lambda \sigma}}{4}\left(\delta g_{\lambda \sigma, \beta} \delta_{\alpha}^{\mu}+\delta g_{\lambda \sigma, \alpha} \delta_{\beta}^{\mu}\right)+\frac{\delta g^{\lambda \sigma}}{4}\left(g_{\lambda \sigma, \beta} \delta_{\alpha}^{\mu}+g_{\lambda \sigma, \alpha} \delta_{\beta}^{\mu}-g_{\lambda \sigma} \varphi_{, \beta} \delta_{\alpha}^{\mu}-g_{\lambda \sigma} \varphi_{, \alpha} \delta_{\beta}^{\mu}\right)\right]
\end{aligned}
$$


Using the fact that ${ }^{(W)} \square g_{\alpha \beta}=g_{\alpha \beta ; \nu}{ }^{; \nu}=g_{\alpha \beta}\left(2 \varphi_{, \lambda} \varphi_{, \rho}+\varphi_{, \rho ; \lambda}\right) g^{\lambda \rho}$, and the expression (29), we obtain that the equation (28) can be written in the form

$$
\begin{aligned}
& \frac{1}{2} \Lambda_{1} g^{\mu \nu}\left[\left(16 \varphi_{, \alpha} \varphi^{, \alpha}-4 g^{\lambda_{1} \rho}\left(2 \varphi_{, \lambda_{1}} \varphi_{, \rho}+\varphi_{, \lambda_{1} ; \rho}\right)\right) \delta g_{\mu \nu}+{ }^{(W)} \square \delta g_{\mu \nu}\right] \\
& ={ }^{(W)} \square \Phi+4\left(2 \varphi_{, \lambda_{1}} \varphi_{, \rho}+\varphi_{, \lambda_{1} ; \rho}\right)\left(W^{\lambda_{1} ; \rho}-2 W^{\lambda_{1}} \varphi^{, \rho}\right)
\end{aligned}
$$

where we have introduced the scalar field $\Phi=W^{\mu} \varphi_{, \mu}$. It can be easily seen that the condition $2 \varphi_{, \mu} \varphi_{, \nu}+\varphi_{, \mu ; \nu}=0$ is valid when the Weyl scalar field $\varphi$ satisfies the formula: $\square \varphi=0$. Thus, when the field $\varphi$ obeys a Riemannian wave equation, the expression (30) reduces to

$$
\frac{1}{2} \Lambda_{1} g^{\mu \nu}\left[16 \varphi_{, \alpha} \varphi^{, \alpha} \delta g_{\mu \nu}+{ }^{(W)} \square \delta g_{\mu \nu}\right]={ }^{(W)} \square \Phi
$$

which must be separated to obtain particular solutions

$$
\begin{aligned}
16|v|^{2} \delta g_{\mu \nu}+{ }^{(W)} \square \delta g_{\mu \nu} & =0, \\
(W) \square \Phi & =0,
\end{aligned}
$$

with $|v|^{2}=\varphi_{, \alpha} \varphi^{\alpha}$ for the Weyl vector field in any point. With the help of the equations (41) and (27), the expression (32) can be separated into a Riemannian part plus a part that depends of the Weyl scalar field in the form

$$
\begin{aligned}
\square \delta g_{\mu \nu} & =10|v|^{2} \Phi, \\
\square \Phi & =2 \Phi_{, \nu} \varphi^{, \nu},
\end{aligned}
$$

which must be viewed as an effective gravitational wave [14] with a massive term originated in the Weyl field. In the case in which $\varphi=0\left(|v|^{2}=0\right)$, the problem reduces to a family of homogeneous equations for Riemannian geometry.

\section{A. Massless Riemannian gravitons}

As was point out in last paragraph, we could obtain an homogeneous Riemannian wave equation in the case with $\varphi=0$, but this is not the only way. We see that (31) directly conduces to

$$
-10 \Phi|v|^{2}+\frac{1}{2} \Lambda_{1} g^{\mu \nu} \square \delta g_{\mu \nu}=\square \Phi-2 \Phi_{, \mu} \varphi^{, \mu}
$$


If we set the particular solution with $\Phi=5 e^{\varphi}$ and $\Phi_{, \mu} \varphi^{\mu}=5 \Phi|v|^{2}$, hence the equation (34), must be rewritten as

$$
\delta g_{k c}=0
$$

plus the condition $\square \varphi=0$ which we already imposed to reduce (30). Notice that the equation (34) takes into account the simplest case corresponding to $\Phi_{, \nu}=0$, which implies $|v|=0$. In this case this expression can be reduced to the particular form of (35), with the additional condition: $\square \Phi=0$.

\section{B. Massless Weyl-like graviton}

To study Weyl massless gravitons, we must take into account (31), with the condition $2 v_{\nu} v_{\mu}+v_{\nu ; \mu}=0$, in order to obtain solutions that make possible ${ }^{(W)} \square \delta g_{a b}=0$, under the corresponding equation for the Riemannian geometry:

$$
\frac{1}{2} g^{\mu \nu(R)} \square \delta g_{\mu \nu}=\left\{-10 \Lambda_{1} g^{\mu \nu}|v|^{2}-2 g_{, \alpha}^{\mu \nu} \varphi^{, \alpha}-2 g^{\alpha \beta} \varphi^{, \gamma}\left(\Gamma_{\beta \gamma}^{\nu} \delta_{\alpha}^{\mu}+\Gamma_{\alpha \gamma}^{\nu} \delta_{\beta}^{\mu}\right)\right\} \delta g_{\mu \nu} .
$$

We see that

$$
g^{\alpha \beta} \varphi^{, \gamma}\left(\Gamma_{\beta \gamma}^{\nu} \delta_{\alpha}^{\mu}+\Gamma_{\alpha \gamma}^{\mu} \delta_{\beta}^{\nu}\right)=-g^{\mu \nu}|v|^{2}-g_{, \mu}^{\mu \nu} \varphi^{, \mu},
$$

which once evaluated in (36), gives us a massive Klein-Gordon equation on the Riemannian manifold

$$
\delta g_{\mu \nu}+f\left(\Lambda_{1}\right)|v|^{2} \delta g_{\mu \nu}=0
$$

with

$$
f\left(\Lambda_{1}\right)=\frac{4-20 \Lambda_{1}}{\Lambda_{1}+1}
$$

It is easy to see that for $\Lambda_{1} \sim \frac{1}{t^{2}}$, we obtain

$$
f\left(\Lambda_{1}\right)_{t \rightarrow \infty} \longrightarrow 4
$$

which in the asymptotic limit for (38)

$$
\bar{\square} \delta g_{\mu \nu}+4|v|^{2} \delta g_{\mu \nu}=0,
$$


corresponding to a massive Riemannian graviton with the mass term originated by the Weyl vector:

$$
m^{2}=4|v|^{2}=4 \varphi_{, \alpha} \varphi^{, \alpha}
$$

It is easy to see that the equations (36) and (31), imply that

$$
{ }^{(W)} \square \delta g_{\mu \nu}=0,
$$

which is linked to a non massive Weyl graviton.

The present formalism admits both kind of solutions: massive and massless nonRiemannian gravitons. This versatility enables us to avoid the problems of massive gravitons by dealing only with effective Riemannian massless gravitons (which comes from a massive non-Riemannian one), and make possible the study of "effective" massive Riemannian gravitons which are really massless in a non-Riemannian sense. In both cases imply a new kind of gravitational waves with source in non-metricity, which is a difference with the usual GR gravitational waves.

\section{GRAVITATIONAL WAVES FROM $\Lambda_{2}$}

In the previous section we have examined gravitational waves in Weyl geometry using (19), which is associated to the third term of (11). Now we shall see that the second term of (11), which also contributes to the total cosmological constant, allows us to construct gravitational waves in a different manner [3]. Although we have supposed that the surface term in (13) is nonzero, we must notice that in general, it is commonly considered in the

literature as null. However, this assertion do not implies that $W_{; \mu}^{\mu}=g_{\mu \nu} W^{\mu ; \nu}$ must be identically zero in the inner of the manifold. Hence, one always can define a geometrical field $\psi_{a b}$, according to

$$
\psi_{\alpha \beta ; \gamma \delta}=\frac{1}{4} g_{\gamma \delta}{ }^{(W)} W_{\alpha ; \beta}
$$

such that

$$
{ }^{(W)} \square \psi_{\alpha \beta}={ }^{(W)} W_{\alpha ; \beta}
$$

This is a wave equation with sources originated in the surface term associated to $\Lambda_{2}$. Using the equations (41) and (26), we obtain

$$
{ }^{(W)} W^{\nu}=\left(\delta\left\{\begin{array}{c}
\nu \\
\mu \rho
\end{array}\right\}-\delta\left\{\begin{array}{c}
\kappa \\
\kappa \rho
\end{array}\right\} \delta_{\mu}^{\nu}\right) g^{\mu \rho}+\left(\delta K_{m r}^{\nu}-\delta K_{\kappa \rho}^{\kappa} \delta_{\mu}^{\nu}\right) g^{m r}=\bar{W}^{\nu}+\Delta W^{\nu},
$$


with $\bar{W}^{\nu}=\left(\delta\left\{\begin{array}{c}\nu \\ \mu \rho\end{array}\right\}-\delta\left\{\begin{array}{c}\kappa \\ \kappa \rho\end{array}\right\} \delta_{\mu}^{\nu}\right) g^{\mu \rho}$ and $\Delta W^{\nu}:=\left(\delta K_{\mu \rho}^{\nu}-\delta K_{\kappa \rho}^{\kappa} \delta_{\mu}^{\nu}\right) g^{\mu \rho}$. This last is null when the generalized contortion tensor is null (i.e. on a Riemannian manifold). Obviously ${ }^{(W)} W_{\alpha ; \beta}=$ $\left(g_{\nu \alpha}{ }^{(W)} W^{\nu}\right)_{; \beta}$. On the other hand, we have

$$
{ }^{(W)} W_{; \nu}^{\nu}=\bar{W}_{\mid \nu}^{\nu}+\Delta W_{\mid \nu}^{\nu}+\bar{W}^{\nu} K_{\nu \mu}^{\mu}+\Delta W^{\nu} K_{\nu \mu}^{\mu}
$$

with $\bar{W}_{\mid \nu}^{\nu}=\phi$ in the equation (10) of [3] , relating the divergence of the Weyl field ${ }^{(W)} W^{\nu}$ with the scalar field employed in such article. In our case it is associated to the Weyl geometry, and the field ${ }^{(W)} W^{\nu}$ must be written in terms of $\Phi=W^{\nu} v_{\nu}$ :

$$
{ }^{(W)} W_{\alpha ; \beta}=\bar{W}_{\alpha \mid \beta}+\left(\Phi_{, \beta}+4 \Phi \varphi_{, \beta}\right) \frac{\varphi_{, \alpha}}{|v|^{2}}
$$

after supposing the Weyl vector field is nonzero. Then, (45) becomes

$$
{ }^{(W)} \square \psi_{\alpha \beta}=\bar{W}_{\alpha \mid \beta}+\left(\Phi_{, \beta}+4 \Phi \varphi_{, b}\right) \frac{\varphi_{, \alpha}}{|v|^{2}},
$$

which clearly reduces to

$$
\bar{\square} \psi_{\alpha \beta}=\bar{W}_{\alpha \mid \beta}
$$

in absence of non-metricity.

\section{MASSLESS GRAVITONS FROM $\Lambda_{1}$ IN PRE-INFLATION}

We consider the case of an expanding universe with cosmological constant $\Lambda_{1} \neq 0$, such that the metric tensor is $[g]_{\mu \nu}=\operatorname{diag}\left[1,-a^{2}(t),-a^{2}(t),-a^{2}(t)\right]$. An interesting case is that of an pre-inflationary universe, with a Hubble parameter

$$
H(t)=\sqrt{\frac{2 \Lambda}{3}} \tanh \left[2 \sqrt{\frac{2 \Lambda}{3}} t\right],
$$

such that the scale factor of the universe is

$$
a(t)=\frac{a_{0}}{\left[1-\tanh ^{2}\left(2 \sqrt{\frac{2 \Lambda}{3}} t\right)\right]^{1 / 4}}
$$

with $a_{0}=\sqrt{\frac{3}{2 \Lambda}}$. This model describes an universe with a Hubble parameter that increases from a null value to an asymptotically constant value $\left.H(t)\right|_{t \gg G^{1 / 2}} \rightarrow \sqrt{\frac{2}{3} \Lambda}$, describing the 
creation of the universe and its transition from a static state to an accelerated de Sitter inflationary expansion. This metric was proposed and studied in a non-metricity free scenario in [3] and we follow his method to solve eq. (35):

$$
\delta g_{k c}=0,
$$

we propose an expansion of the form

$$
\begin{aligned}
\delta g_{a b}(t, \vec{x}) & =\frac{1}{(2 \pi)^{3 / 2}} \sum_{M=+, \times} \int d^{3} k e_{a b}^{M}(\hat{z}) \\
& \times\left[A_{k} e^{i \vec{k} . \hat{z}} \chi_{c}(t)+A_{k}^{\dagger} e^{-i \vec{k} . \hat{z}} \chi_{c}^{*}(t)\right],
\end{aligned}
$$

where index $M=+, \times$ denote the transverse polarizations,$+ \times$, on the plane normal to $\vec{k}$ and $e_{a b}^{M}$ are the components of the polarization tensor, such that $e_{a b}^{M} \bar{e}_{M^{\prime}}^{a b}=\delta_{M^{\prime}}^{M}$. We work in the frame in which $\vec{k}$ is in the $\hat{z}$ direction, and the polarizations are

$$
e_{a b}^{+}=\left(\begin{array}{cc}
1 & 0 \\
0 & -1
\end{array}\right)_{a b}, \quad e_{a b}^{\times}=\left(\begin{array}{cc}
0 & 1 \\
1 & 0
\end{array}\right)_{a b},
$$

with $a, b$ spanning the $(x, y)$ plane. We shall use the TT gauge in order to solve the gravitational waves equations, such gauge is represented by next conditions

$$
\delta g_{0 \mu}=0, \quad \delta g_{i}^{i}=0, \quad \bar{\nabla}^{j} \delta g_{i j}=0
$$

The equation of motion for the modes $\chi_{k}(t)$ is

$$
\ddot{\chi}_{c}(t)+3 \frac{\dot{a}}{a} \dot{\chi}_{c}(t)+\frac{k^{2}}{a(t)^{2}} \chi_{c}(t)=0 .
$$

The annihilation and creation operators $A_{k}$ and $A_{k}^{\dagger}$ satisfy the usual commutation algebra

$$
\left[A_{k}, A_{k^{\prime}}^{\dagger}\right]=\delta^{(3)}\left(\vec{k}-\overrightarrow{k^{\prime}}\right), \quad\left[A_{k}, A_{k^{\prime}}\right]=\left[A_{k}^{\dagger}, A_{k^{\prime}}^{\dagger}\right]=0
$$

We obtain the normalization condition for the modes $\chi_{c}(\tau)$ :

$$
\chi_{c}(t) \frac{d \chi_{c}^{*}(\tau)}{d \tau}-\chi_{c}^{*}(t) \frac{\chi_{c}(\tau)}{d \tau}=i\left(\frac{a_{0}}{a(\tau)}\right)^{3},
$$

with $\tau=b t$, where $b=\sqrt{\frac{2 \Lambda}{3}}=\frac{1}{a_{0}}$. The asterisk in (58) denotes the complex conjugated. For the case in which the Hubble parameter and the scale factor are given respectively by 
(51) and (52), the general solution for the amplitudes $\chi_{c}(\tau)$ is

$$
\begin{aligned}
\chi_{c}(\tau) & =C_{1} \frac{\sinh (\tau)}{\sqrt{2 \cosh ^{2}(\tau)-1}} \\
& \times \operatorname{Hn}\left[-1, \frac{c^{2}-1}{4} ; 0, \frac{1}{2}, \frac{3}{2}, \frac{1}{2} ;-\tanh ^{2}(\tau)\right] \\
& +C_{2} \frac{\cosh (\tau)}{\sqrt{2 \cosh ^{2}(\tau)-1}} \\
& \times \operatorname{Hn}\left[-1, \frac{c^{2}+1}{4} ;-\frac{1}{2}, 0, \frac{1}{2}, \frac{1}{2} ;-\tanh ^{2}(\tau)\right]
\end{aligned}
$$

where $\operatorname{Hn}[a, q ; \alpha, \beta, \gamma, \delta ; z]=\sum_{j=0}^{\infty} c_{j} z^{j}$ is the Heun function. We can do a series expansion in both sides of (158) according to

$$
\chi_{c}(t) \frac{d \chi_{c}^{*}(\tau)}{d \tau}-\chi_{c}^{*}(t) \frac{\chi_{c}(\tau)}{d \tau}-i\left(\frac{a_{0}}{a(\tau)}\right)^{3}=\sum_{N=1}^{\infty} f_{N}(c) \tau^{N}=0
$$

where $f_{N}\left(c_{n}^{(N)}\right)=0$, for each $N$. There are $2 N$ modes for each $N$-th order of the expansion, we obtain conditions for coefficients $C_{1}$ and $C_{2}$, and the values for wave-number $k$. From the zeroth order expansion (in $\tau$ ), we obtain that $C_{2}=i C_{1} / 2$. Hence, we shall choose $C_{1}=1$ and $C_{2}=i / 2$.

The serie is infinite so that there are infinite values of quasi-normal modes with projection on the plane orthogonal to the direction of propagation with zero norm according to $\hat{z}$ : $\vec{k}=c_{n}^{(N)} \hat{e^{1}}+\left( \pm i c_{n}^{(N)}\right) \hat{e^{2}}+k \hat{e^{3}}$, with complex values $c_{n}^{(N)}$ which provide us the quantization of tensor fields $\delta g_{a b}$. Notice that $\|\vec{k}\|^{2}=k^{2}$, so that the norm of the polarization vectors on the plane $\left(\hat{e^{1}}, \hat{e^{2}}\right)$, is zero. When the polarization modes which are purely imaginary or purely reals correspond to values with polarization + . On the other hand, modes with complex- $c_{n}^{(N)}$ correspond to those with polarization $\times$. In the table we have included the square wave-numbers for the first five orders of the expansion: 


\begin{tabular}{|l|lr|l|l|l|}
\hline Square wavenumbers $c_{n}^{(N)}$ & $\left(c_{n}^{(N)}\right)^{2}$ values \\
\hline$\left(c^{(1)}\right)^{2}$ & -0.5 & -0.57 & \\
\hline$\left(c^{(2)}\right)^{2}$ & 6.57 & $0.01+1.25 \mathrm{i}$ & $0.01-1.25 \mathrm{i}$ & \\
\hline$\left(c^{(3)}\right)^{2}$ & -53.78 & -1.29 & $9.85+9.40 \mathrm{i}$ & $9.85-9.40 \mathrm{i}$ & \\
\hline$\left(c^{(4)}\right)^{2}$ & 9.60 & 12.72 & -1.34 & $13.84+18.30 \mathrm{i}$ & $13.84-18.30 \mathrm{i}$ \\
\hline$\left(c^{(5)}\right)^{2}$ & 5.93 & & &
\end{tabular}

which is provided in [3] and formally solves eq. (35). We must remark that although eq. (35) is formally equal to eq. (42) in [3] the origin of our equation is slightly different since we got eq. (35) as the expression for an effective gravitational wave from a Weyl space-time which directly describes the tensorial perturbation of a background FRW metric.

\section{FINAL COMMENTS}

We have studied a new formalism to describe gravitational wave dynamics on a Weylian manifold. The case in which the geometry is free of torsion has been examined. This case will be considered in a further work. Of course, due to the fact the nature of the manifold is Weylian, the non-metricity and the boundary terms must be taken into account. The fist case was studied in Sect. III and provide us an integrable treatment of the Weylian manifold. The second case was studied in Sect. IV and is important because nontrivial boundary terms could be responsible for the cosmological constant produced by a source inside a 3D Gaussian-like hypersurface that encloses that source. This source also could be responsible for the production of gravitational waves. To finalize, it is important to notice that in the paper [15], the authors obtain a gauge-invariant relativistic quantum geometry by using a Weylian-like manifold with a geometric scalar field, which provides a gauge-invariant relativistic quantum theory, in which the quantum algebra of the Weylian-like field depends of the observers. Is important to notice that, in the context of [15], the fields ${ }^{(W)} W_{\alpha}$ and $\bar{W}_{\alpha}$ must be interpreted as variations, the first one linked to the quantum geometry described by the Weylian-like manifold, and the second one related to classical process related to the Riemannian manifold, which must be zero. In order to make our formulation compatible with the ideas of such work, we must impose the condition $\bar{W}_{\alpha}=0$, which conduces from 
(48) and (49), to

$$
{ }^{(W)} W_{\alpha ; \beta}=\left(\Phi_{, \beta}+4 \Phi \varphi, \beta\right) \frac{\varphi_{, \alpha}}{|v|^{2}}={ }^{(W)} \square \psi_{\alpha \beta},
$$

where the last equation represents an effect originated in the $\Lambda_{2}$ frontier contribution and enterally due to non-metricity, which is of quantum nature.

In Sct. (VI), we have solved the particular example of the gravitational wave associated to Riemannian massless gravitons over a FRW background describing a pre-inflationary scenario. The normalization conditions for the modes impose that the wavenumbers for $\delta g_{\alpha \beta}$ are an infinity number of discrete complex values for the polarization modes, which can be real and imaginary for the + -modes and complex for the $\times$-modes.

\section{Acknowledgements}

J. M. Romero and M. Bellini acknowledge UNMdP and CONICET for financial support. J.E.M.A acknowledges CONACYT México, Centro Universitario de Ciencias Exactas e Ingenierias and Centro Universitario de los Valles, of Universidad de Guadalajara for financial support.

[1] J. W. York, Phys. Rev. Lett. 16: 1082 (1972).

[2] G. W. Gibbons, S. W. Hawking, Phys. Rev. D10: 2752 (1977).

[3] L. S. Ridao, M. Bellini, Astrophys. Space Sci. 357: 94 (2015).

[4] D. Hilbert, Die Grundlagen der Physik, Konigl. Gesell. d. Wiss. Gttingen, Nachr. Math.-Phys. Kl. 395-407 (1915);

D.D. Sokolov, Cosmological constant, in Hazewinkel, Michiel, Encyclopedia of Mathematics, Springer, ISBN 978-1-55608-010-4 (2001);

S. M. Carroll, "Spacetime and Geometry", Addison Wesley, ISBN 0-8053-8732-3 (2004).

[5] M. Nakahara, Geometry, Topology and Physics, IoP, Bristol Philadephia, ISBN 075030606 8 (2003);

Ø. Grøn, S. Hervik, Einstein's General Theory of Relativity, Springer, ISBN-13:978-0-38769199-2 (2007). 
[6] S. Kopeikin, M. Efroimsky, G. Kaplan, Relativistic Celestial Mechanics of the Solar System, John Wiley and Sons, ISBN 9783527408566 (2011).

[7] É.Cartan, Sur les varits connexion affine, et la thorie de la relativit gnralise (premire partie), Annales Scientifiques de l'cole Normale Suprieure 40: 325412 (1923);

F.W. Hehl, P. von der Heyde, G.D. Kerlick, J.M. Nester, Rev. Mod. Phys. 48: 393 (1976);

M. Spivak, A comprehensive introduction to differential geometry, Vol. II, Houston, Texas: Publish or Perish, ISBN 0-914098-71-3 (1999).

[8] S. Kobayashi, K. Nomizu, Foundations of Differential Geometry, Vols. 1 and 2 (New ed.), Wiley-Interscience, ISBN 0-471-15733-3 (1996). (2007).

[9] M. Israelit, Gen. Rel. Grav. 29: 1597 (1997);

M. Nakahara, Geometry, Topology and Physics, IoP, Bristol Philadephia, ISBN 075030606 8 (2003);

M. Israelit, Found. Phys. 35: 1769 (2005).

[10] H.Kleinert, Gauge Fields in condensed matter, p.1427, World Scientific, Singapur (1989);

H. Kleinert, Path Integrals in Quantum Mechanics, Statistics, Polymer Physics, and Financial Markets, p.786, 5th edition, pp. 1-1547, World Scientific, Singapore (2009). Presented text is a free translation of the spanish version.

[11] H. Kleinert, Path Integrals in Quantum Mechanics, Statistics, Polymer Physics, and Financial Markets, Chapt. 10, 5th edition, pp. 1-1547, World Scientific, Singapore (2009).

[12] J. M. Romero, M. Bellini, Eur. Phys. J. C74: 3043 (2014);

J. M. Romero, M. Bellini, Can. J. Phys. 93: 445 (2014);

[13] M. Novello, H. Heintzmann, Phys. Lett. A98: 10 (1983);

C. Romero, J. B. Fonseca-Neto and M. L. Pucheu, Class. Quant. Grav. 29: 155015 (2012).

[14] I. Chakrabarty, Gravitational Waves: An Introduction, E-print arXiv:physics/9908041 (1999);

J. Weber, General Relativity and Gravitational Waves, Dover ed. p. cm. (2004);

K. Hinterbichler, Rev. Mod. Phys. 84: 671 (2012);

S. Capozziello, M. De Laurentis, M. Paolella, G. Ricciardi, Int. J. Geom. Meth. Mod. Phys. 12: 1550004 (2015).

[15] L.S. Ridao, M. Bellini, Phys. Lett. B751 : 565 (2015). 